\title{
Transfer of conditional reasoning: Effects of explanations and initial problem types
}

\author{
PAUL A. KLACZYNSKI \\ West Virginia University, Morgantown, West Virginia \\ HAROLD GELFAND \\ St. Bonaventure University, St. Bonaventure, New York \\ and \\ HAYNE W. REESE \\ West Virginia University, Morgantown, West Virginia
}

\begin{abstract}
Transfer of reasoning on Wason's (1966) selection task was explored in three experiments. Experiment 1 tested the effects of problem explanations and verbalization instructions on transfer from abstract or thematic problems to abstract problems. Explanations facilitated transfer only when the initial problems were abstract; verbalization did not produce transfer between problems. Experiment 2 explored the effects of problem similarity and explanations on transfer between problems. Although transfer occurred following explanations, no effect of similarity was found for thematic problems. In both of these experiments, the thematic effect (Wason \& Shapiro, 1971) was observed. Experiment 3 examined the effects of explanations to abstract or thematic problems on transfer to subsequent abstract or thematic problems. Transfer of reasoning occurred from both initial problem types, particularly to problems of the same type; however, transfer occurred to a greater extent from abstract problems than from thematic problems. The results are discussed in terms of problem similarity and Cheng and Holyoak's (1985) pragmatic reasoning schema hypothesis.
\end{abstract}

\section{Reasoning with Abstract Conditionals}

Research aimed at discerning the processes of deductive reasoning has relied heavily on Wason's (1983) fourcard selection task. Briefly, this task consists of "if $p$, then $q$ " rules that participants are told may or may not be true. An example of an abstract form of such a rule is, "If there is an A on one side of a card, then there must be a 4 on the other side." An array of four cards is then presented representing " $p$ " " $q$ " "not $p$ " and "not $q$." In the example, these cards could be " $A$," " 4 ," " $D$," and " 7, , respectively. Participants are told that each card has a number on one side and a letter on the other side. The task is to choose only those cards that will allow the participants to decide with certainty whether the rule is true or false. The correct solution is selection of both the " $p$ " (A) and the "not $q$ " (7) cards. Only this combination can falsify the rule; that is, if the affirmation of the antecedent $(p)$ and the denial of the consequent (not $q$ ) appeared on the same card, then the rule would be false.

Wason's $(1966,1968)$ original results-and subsequent replications (e.g., Evans, 1972, 1977; Manktelow \& Evans, 1979)-showed that almost all participants select the " $p$ " card and the majority also select the " $q$ " card. Fewer than $10 \%$ make the correct selection of " $p$ " and "not $q$ "; this finding suggests that in reasoning about these problems, most persons do not use the rules of for-

Address correspondence to Paul A. Klaczynski, Department of Psychology, West Virginia University, Morgantown, WV 26506-6040. mal logic. Valentine (1985) showed that the type of errors found on the selection task varies according to the type of instructions given. Instructions to test whether a rule was violated resulted in a large number of verification errors, that is, choices of " $p$ " and " $q$ "; however, instructions to determine whether the rule is true or false resulted in increased variability in card selection. This finding further illustrates the difficulty participants have with this type of reasoning problem.

\section{Reasoning with Thematic Conditionals}

The complexity of explanation is increased by the findings from research with a modified version of the selection problem. Wason's $(1966,1968)$ original research involved rules containing arbitrary relations between the antecedent and consequent (in the relevant literature, these rules are designated as abstract). Several early studies indicated that when the task was modified to incorporate realistic or thematic content rather than abstract content, performance improved dramatically (Bracewell \& Hidi, 1974; Gilhooly \& Falconer, 1974; Johnson-Laird, Lengrezi, \& Lengrezi, 1972; Van Duyne, 1974; Wason \& Shapiro, 1971). In the thematic version of the task, the rule referred to concrete materials and a familiar relation between the antecedent and the consequent. For example, "If I go to Manchester, I travel by car" (Wason \& Shapiro, 1971).

Further extending these findings, Griggs and Cox (1982) found better performance when the rule used was 
directly related to the past experiences of participants Several investigators (e.g., Manktelow \& Evans, 1979; Reich \& Ruth, 1982; Yachanin \& Tweney, 1982), however, found no evidence of superior reasoning with thematic materials, although the rules used in these studies do not appear to be as related to the past experiences of participants as that of Griggs and Cox (1982).

Griggs (1983) offered a memory-cuing explanation of the effect of familiarity. According to this explanation, thematic problems improve performance if they allow participants to recall past experience with the problem content, the rule presented, and a counterexample of the rule. A similar explanation was proposed by Manktelow and Evans (1979). Subsequent research (Griggs, 1984) has provided some support for the memory-cuing explanation, although the cuing effect of thematic materials appears to be partially mediated by instructions: Instructions to test rule violations enhance the effect of thematic content, whereas instructions to determine the truth or falsity of the rule diminish-but do not extinguish-the effect (Chrostowski \& Griggs, 1985).

In a series of investigations, Cheng and Holyoak (1985; Cheng, Holyoak, Nisbett, \& Oliver, 1986) have provided compelling evidence for an alternative explanation. They suggested that in the course of everyday experiences, persons induce generalizable reasoning structures. These pragmatic reasoning schemas extend beyond formal logic not only to determine the inferences about rules that are useful, but also to interpret nonlogical premises involving permissions, obligations, and causation. Abstract problems are particularly difficult because they are not tied to experience and do not evoke a reasoning schema. In contrast, a permission schema is evoked by some thematic problems. The permission schema contains a set of rules regarding the preconditions that must be met before a certain action can take place. These rules operate consistently with the rules of formal logic; therefore, problems that evoke this schema are solved correctly. However, other thematic problems may evoke schemas containing rules that do not correspond closely to those of formal logic, precluding correct solutions.

\section{Transfer of Reasoning Between Problem Types}

Understanding of the mechanisms involved in deductive reasoning may be furthered by determining whether, and under what conditions, the reasoning used to solve thematic problems transfers to problems of other types. Various investigators, including Johnson-Laird et al. (1972) and Lunzer, Harrison, and Davey (1972), have found that participants' reasoning on thematic problems did not transfer to abstract problems. A related study by Cox and Griggs (1982) extended these findings; although no evidence of transfer from thematic to abstract problems was found, significant transfer did occur between thematic problems. The thematic rule used in this study was, "If a person is drinking beer, then the person must be over 19." The analogous, but not familiar rule was, "If a person is wearing blue, then the person must be over 19."
Cheng et al. (1986) found that training in formal logic produced transfer to other selection problems with different content. In a separate study, Cheng et al. found that when participants interpreted the rules presented in $a b$ stract problems as obligations (e.g., if condition $\mathrm{A} \mathrm{oc}$ curs, then obligation $B$ arises), previous training on a general obligation schema improved performance.

Berry (1983) found that reasoning transferred from thematic to abstract problems when participants were given minimal explanations of thematic problems in an initial set and were instructed to verbalize the reasoning behind their answers to each initial problem. This study provided the immediate impetus for the present research, and therefore Berry's design bears detailed examination.

Berry (1983) included five conditions: three thematic conditions and two abstract conditions. In two of the thematic conditions, a minimal explanation was given to participants after the first problem, followed by instructions to verbalize (either during or immediately after problem solving) their reasoning on each of four problems. Participants were then tested for transfer on a set of abstract problems. The third thematic condition was similar to the first two except that no verbalization instructions were given. In the abstract conditions, unlike the thematic conditions, participants were not given a final set of problems on which they could be tested for transfer. In one abstract condition, participants received a minimal explanation after the first problem, but instructions to verbalize were not given. The other abstract condition served as a control in which neither the minimal explanation nor the instruction to verbalize was given.

Berry (1983) found transfer from thematic to abstract problems in the two verbalization conditions, and no transfer when only the minimal explanation was presented. Unfortunately, Berry's design left the source of the transfer unclear; that is, although minimal explanations were apparently not sufficient for transfer to occur, the observed facilitation by verbalization was confounded with explanations in both thematic conditions in which verbalization instructions were given. Also, the effect of verbalization on transfer between sets of abstract problems is unknown. Finally, the type of thematic problems used by Berry may have influenced her findings; that is, the rules (e.g., "If the flavor of the jam is plum, then it has a $2 p$ sticker on it") may not have tapped participants' past experiences and, therefore, seem to be more similar to abstract problems. Chrostowski and Griggs (1985) reported no facilitative effect of verbalization instructions on performance, further compounding this issue.

In the present study, three experiments were conducted to provide a clearer understanding of the conditions under which reasoning transfers between problems on the selection task. Another purpose of this research was to explore participants' performance on abstract versus thematic problems. However, in contrast to much of the previous research, participants received several abstract or thematic problems. Assessing performance across a se- 
ries of problems has the advantage of revealing participants' typical performance, as well as variations in their performance.

\section{EXPERIMENT 1}

The primary purpose of Experiment 1 was to clarify the findings of Berry (1983) regarding the effects of problem explanations and verbalization instructions. These variables were manipulated during an initial set of either abstract or thematic problems, and effects on transfer to abstract problems were assessed. The predictions were as follows: (1) When no explanations are given during the initial set, performance will be better on thematic than on abstract problems. (2) Based on Berry's results, transfer from thematic to abstract problems will occur when participants are given explanations of thematic problems along with instructions to verbalize the reasoning behind their selections; however, verbalization instructions alone will not be sufficient to produce transfer to abstract problems. (3) Transfer to a final set of abstract problems will occur when explanations are given for an initial set of abstract problems, but not when the initial set is thematic. This prediction was based on the finding (Cox \& Griggs, 1982) that transfer occurred between analogous problems. After hearing explanations to several abstract problems, participants should make the necessary analogy to solve the remaining abstract problems.

\section{Method}

\section{Subjects}

The participants were 64 students recruited from introductory psychology classes at St. Bonaventure University. Each participant was randomly assigned to one of eight experimental conditions and tested individually in a $1 / 2-h$ session.

\section{Tasks and Procedure}

Nine abstract and five thematic problems were used. The abstract problems were similar to the original rule used by Wason (1966). All abstract problems contained instances of abstract concepts or categories in the antecedent and consequent with no familiar theme connecting them. Examples of abstract problems appear in Appendix $\mathrm{A}$.

The thematic problems were modeled on that of Griggs and Cox (1982) and were designed to be rules that were familiar to the participants. With the exception of the "drinking beer" problem (Griggs \& Cox, 1982), all problems were developed by the first author. These also are presented in Appendix A.

Each problem included four selection alternatives, corresponding to affirmation of the antecedent $(p)$, affirmation of the consequent $(q)$, denial of the antecedent (not $p$ ), and denial of the consequent (not $q$ ). Within each problem, these alternatives were presented in counterbalanced order across participants. The correct answer to each problem was selection of the two alternatives that could falsify the rule, that is, " $p$ " and "not $q$."

The experiment was a $2 \times 2 \times 2$ between-subjects factorial design. The independent variables were initial problem type (abstract vs. thematic), explanation (given vs. not given), and verbalization instructions (given vs. not given). Participants were told that they would be given a series of nine reasoning problems, that each of these problems involved a rule that could be true or false, and that their task on each problem was "to decide which card or cards you would turn over in order to decide whether or not the rule you are given is in effect." After these instructions had been read, participants were given a booklet containing nine problems, one per page.

In the abstract conditions, the first five problems were abstract; in the thematic conditions, the first five problems were thematic. Within each condition, the order of these initial problems was counterbalanced across participants. In all conditions, the final four problems were abstract. These final four problems were the same in each condition and were presented in counterbalanced order across participants.

In the explanation conditions, participants were read in-depth explanations of the correct and incorrect selections immediately after they completed each of the first five problems. Examples of explanations to both abstract and thematic problems are presented in Appendix $A$. In the verbalization conditions, participants were told to think aloud during each of the first five problems.

\section{Scoring}

Two systems were used to score participants' selections. In the initial problem set, only problems 2-5 were included in the scoring because in the explanation conditions, the first explanation was given only after the response to the first problem. The first scoring system was based on fully adequate ( $p$ and not $q$ ) selections only. Participants received a score of 1 for correct solutions and 0 for incorrect solutions. Therefore, on both the initial and the final problem sets, scores could range from 0 to 4 . In the second scoring system, participants' selections were classified according to predominant selection patterns. Participants responding with the same selection patterns on at least three of the four problems in a set were scored 1 for that pattern and 0 for all other patterns. If no selection pattern was predominant (i.e., if a participant did not respond the same way more than twice), the participant was scored in an "unclassifiable" category and given a 0 for all other selection patterns.

In much of the previous research in this area, mean numbers of selections of individual cards were analyzed. However, means per card reveal little, if anything, about patterns of selections of individual participants. Therefore, means per card are not reported herein. (Interested readers may obtain analyses of mean selections per card upon request from the first author.)

\section{Results}

\section{Initial Problems}

Correct selections. A $2 \times 2 \times 2$ (initial problem type $\times$ explanation $x$ verbalization instructions) analysis of variance was conducted on the correct selections in the initial problem set. The only statistically significant effects were the explanation main effect and the problem type $\times$ explanation interaction $[F(1,56)=32.34, p<.001$; $F(1,56)=11.98, p=.001$, respectively $]$. Verbalization had no significant main effect $[F(1,56)=2.20, p=$ $.140]$, and did not interact significantly with either initial problem type or explanation $(F<1)$. The relevant means are shown in the upper left portion of Table 1.

Further analyses showed that in the explanation conditions, performance did not differ significantly on the abstract and thematic problems $[F(1,28)=1.14, p=.295]$, but in the no-explanation conditions, performance was significantly better when the initial problems were thematic than when they were abstract $[F(1,28)=12.80, p=$ $.002]$. Thus, the first prediction was supported. The magnitude of this thematic effect $(43 \%$ correct on the initial 
Table 1

\begin{tabular}{|c|c|c|c|c|}
\hline \multirow[b]{3}{*}{ Initial Problems } & \multicolumn{4}{|c|}{ Problems } \\
\hline & \multicolumn{2}{|c|}{ Initial } & \multicolumn{2}{|c|}{ Final } \\
\hline & Explanation & No Explanation & Explanation & No Explanation \\
\hline \multicolumn{5}{|c|}{ Correct Selections Only* } \\
\hline Abstract & 2.82 & .50 & 3.56 & .56 \\
\hline Thematic & 2.38 & 1.80 & .38 & .12 \\
\hline \multicolumn{5}{|c|}{ Predominant Selection Patterns $\dagger$} \\
\hline \multicolumn{5}{|l|}{ Abstract } \\
\hline$p$, not $q$ & .63 & .06 & .88 & .13 \\
\hline$p, q$ & .00 & .38 & .06 & .50 \\
\hline Other & .00 & .06 & .06 & .31 \\
\hline Unclassifiable & .37 & .50 & .00 & .06 \\
\hline \multicolumn{5}{|l|}{ Thematic } \\
\hline$p, \operatorname{not} q$ & .42 & .31 & .13 & .00 \\
\hline$p, q$ & .07 & .00 & .43 & .50 \\
\hline Other & .00 & .06 & .25 & .31 \\
\hline Unclassifiable & .50 & .63 & .19 & .19 \\
\hline
\end{tabular}

thematic problems, $6 \%$ correct on initial abstract problems), however, was not as great as others have observed (e.g., Johnson-Laird et al., 1972; Wason \& Shapiro, 1971).

Predominant selection patterns. Inspection of patterns of predominant selections during the initial problem set revealed that only two patterns occurred frequently enough to warrant inclusion in separate categories. These patterns were " $p, q$ " and " $p$, not $q$." All other patterns were placed in either an "other" category or an "unclassifiable" category. The overall analysis again indicated that verbalization, either alone or in combination with other variables, had no effect on performance. It was therefore dropped from further analyses. A $2 \times 2 \times 4$ (initial problem type $\times$ explanation $\times$ selection pattern) analysis of variance, with selection pattern as a within-subjects variable, revealed a significant three-way interaction $[F(3,180)=$ $3.25, p=.023]$. This interaction was followed up by separate analyses for each explanation condition.

Explanation conditions. In the explanation conditions, the initial problem type $\times$ selection pattern interaction was significant $[F(3,90)=3.44, p=.020]$. As can be seen in the lower left portion of Table 1 , the " $p$, not $q$ ", and "unclassifiable" selection patterns were the most frequent selection patterns in both types of initial problems, but the relative proportions of the selections differed according to problem type.

No-explanation conditions. In the no-explanation conditions, the initial problem type $\times$ selection pattern interaction was also significant $[F(3,90)=5.85, p=.001]$, but it reflected a different pattern of results. When initial problems were abstract, the " $p, q$ " selection pattern was more frequent than " $p$, not $q$ " $[F(1,45)=11.30, p=$ $.002]$ and "other" selection patterns $[F(1,45)=11.30$, $p=.002]$. "Unclassifiable" patterns were also more frequent than " $p$, not $q$ " $[F(1,45)=21.30, p<.001]$ and "other" selection patterns $[F(1,45)=21.30, p<.001]$. In contrast, when initial problems were thematic, the " $p$, not $q$ " selection pattern was more frequent than the " $p$, $q$ ' $[F(1,45)=10.64, p=.003]$ and "other'" selection patterns $[F(1,45)=6.92, p=.002]$, but was less frequent than "unclassifiable" patterns $[F(1,45)=11.30$, $p=.002]$.

Comparisons between the selection patterns of participants in the abstract and thematic conditions showed that " $p$, not $q$ " - the correct selection pattern-was more frequent in the thematic condition $[F(3,45)=10.64, p=$ $.001]$, and " $p, q$ " was more frequent in the abstract condition $[F(3,45)=16.00, p<.001]$. Thus, the thematic effect was again observed.

\section{Transfer}

Correct selections. A $2 \times 2 \times 2$ (initial problem type $\times$ explanation $\times$ verbalization instructions) analysis of variance was used to assess transfer to the final abstract set. Verbalization during the initial set of problems did not significantly affect reasoning on the final set of problems $[F(1,56)<1]$, and verbalization did not interact significantly with any other variable [largest $F(1,56)=.90$ ] The second prediction, then, was not supported: verbalization instructions alone did not produce transfer, and verbalization and problem explanations did not interact to produce transfer.

The initial problem type $\times$ explanation interaction was significant $[F(1,56)=29.88, p<.001]$, as were the main effects of initial problem type $[F(1,56)=44.26, p<$ $.001]$ and explanation $[F(1,56)=35.28, p<.001]$. The next step taken in the analysis was to determine the nature of the initial problem type $x$ explanation interaction.

The upper right portion of Table 1 shows the relevant means. As can be seen, transfer to a final set of abstract problems occurred only when explanations were given to 
participants during an initial set of problems that were also abstract, confirming the third prediction. Performance in this condition was significantly better than after initial abstract problems without explanations $[F(1,28)$ $=46.08, p<.001]$, and significantly better than after thematic problems with explanations $[F(1,28)=77.48$, $p<.001]$ or without explanations $[F(1,28)=64.32$, $p<.001]$. Performance did not differ significantly across the latter three conditions [largest $F(1,28)=1.05$ ]

Predominant selection patterns. A $2 \times 2 \times 4$ (initial problem type $\times$ explanation $\times$ selection pattern) anal$y$ sis of variance was conducted on predominant selection patterns in the final problem set. The three-way interaction was highly significant $[F(3,180)=6.70, p<.001]$. The data were therefore analyzed separately by initial problem type.

Initial problems abstract. For abstract problems, the explanation $\times$ selection pattern interaction was significant $[F(3,90)=12.01, p<.001]$. In the abstract conditions with explanations, the " $p$, not $q$ " selection pattern was more frequent than any other pattern [smallest $F(1,90)=53.59, p<.001]$. With no explanations, " $p$, $q$ " was selected more often than " $p$, not $q$ " $[F(1,90)$ $=10.90, p=.002]$, "other" patterns $[F(1,90)=4.30$, $p=.039]$, and "unclassifiable" selection patterns $[F(1,90)=15.70, p<.001]$. Between-condition comparisons showed that the " $p$, not $q$ " selection pattern was more frequent in the explanation than in the no-explanation condition $[F(1,90)=61.6, p<.001]$, and " $p, q$ " was more frequent in the no-explanation condition than in the explanation condition $[F(1,90)=27.37, p<.001]$. The means for these selection patterns are presented in the lower right section of Table 1.

Initial problems thematic. In the conditions with initial thematic problems, the explanation $\times$ selection pattern interaction was nonsignificant $(F<1)$, but the main effect of selection pattern was significant $[F(3,90)=5.36$, $p=.002$ ]. Further analyses showed that " $p, q$ " was more frequent than either the " $p$, not $q$ " $[F(1,90)=12.80$, $p<.001]$ or the "unclassifiable" selection pattern $[F(1,90)=4.90, p=.028]$, but was not significantly more frequent than the "other" selection pattern $[F(1,90)$ $=2.89, p=.09$ ].

Explanation conditions. In the conditions with explanations, the " $p$, not $q$ " selection pattern occurred more frequently in the abstract conditions $[F(1,90)=72.47$, $p<.001]$, and the " $p, q$ " selection pattern occurred more frequently in the thematic conditions $[F(1,90)=$ $24.24, p<.001]$.

No-explanation conditions. In the conditions with no explanations, no significant differences were found between the selection patterns following initial abstract problems and those following initial thematic problems [largest $F(1,90)=2.34, p=.123$ ].

Both scoring systems, thus, revealed that transfer to a final set of abstract problems occurs only when explana- tions are given to an initial set of problems that are also abstract.

\section{Discussion}

The results of Experiment 1 failed to replicate the findings of Berry (1983). Verbalization, either alone or in combination with problem explanations, did not facilitate transfer of reasoning from either thematic or abstract problems to abstract problems. This failure to replicate Berry's results may have occurred because the thematic problems used by Berry were probably not as closely related to the past experiences of participants as were the thematic problems used in the present study; however, not all participants fully complied with the verbalization instructions, a problem also encountered by Chrostowski and Griggs (1985).

Experiment 1 did, however, reveal that when participants were given in-depth explanations after each initial problem in an abstract set, transfer occurred to a final set of abstract problems. Consistent with prior research (e.g., Lunzer et al., 1972; Wason \& Shapiro, 1971), no transfer occurred from thematic to abstract problems, even when explanations were given during the initial thematic set. Finally, in the no-explanation conditions, participants' performance was better when the initial set was thematic than when it was abstract (i.e., the controversial thematic effect was observed).

A contextual similarity hypothesis was developed to account for these various effects. According to this hypothesis, the "context" of a problem consists of related elements from past experience (e.g., events from past experience) or from the problem itself (e.g., words or phrases from the problem). Similar problems have overlapping contexts. The greater the number of shared elements, the greater the similarity (i.e., contextual overlap) between problems. The more similar two problems are, the greater the likelihood that the type of reasoning used on the first problem will be transferred to the second problem.

On the assumption that abstract problems are novel to the participants, the context of each of these problems consists only of the elements presented in the problem. Also, because any abstract problem differs from any other abstract problem only in the different words used for " $p$," " $q$," "not $p$," and "not $q$," these problems are very similar, and reasoning (however incorrect) should readily transfer between them. Thematic problems, in contrast, often exist in contexts that are dissimilar to those of other problems because the elements in them are primarily tied to elements from past experience. Therefore, reasoning is less likely to transfer from thematic problems to other thematic problems or to abstract problems.

When an explanation of one abstract problem is given, it adds elements to the context of that problem that are relevant to the solution of that problem. Because (1) the context of this problem contains not only the elements 
presented in the problem but also elements presented with the explanation and (2) the reasoning associated with the new context should transfer to other abstract problems, participants are now able to solve further abstract problems.

Typical thematic problems are logically, but not contextually, similar. Therefore, even when the elements from an explanation are incorporated into the context of a thematic problem, reasoning does not transfer to further problems. The thematic effect may occur because elements presented in the problem may be associated with elements from past experience that enable reasoning that was used in the past to transfer to the current problem. This comparison, however, takes place within the context of a given thematic problem.

\section{EXPERIMENT 2}

The purpose of Experiment 2 was to test predictions deduced from the context/reason-by-analogy hypothesis. Initial and transfer problems were presented with or without explanations. These problems were either both abstract or both thematic, and those in the thematicthematic pairs were either similar or dissimilar in context. The specific predictions were:

1a. Following an explanation, transfer will occur between two abstract problems because the contexts of any two abstract problems are very similar. Thus, more transfer will occur between abstract problems given with explanations than between thematic problems given with explanations.

1b. After an explanation for a thematic problem, the likelihood of solving a subsequent thematic problem will be greater if the latter problem is similar (i.e., contextually related) rather than dissimilar to the problem for which the explanation was given.

2a. Without explanations, a thematic effect will again be found; that is, performance will be better on abstract than on thematic problems.

2b. Without explanations, performance will be better on the second thematic problem of a pair when the initial problem is similar rather than dissimilar to the transfer problem.

\section{Method}

\section{Subjects}

The participants were 96 students from introductory psychology classes at St. Bonaventure University. All participants were randomly assigned across six experimental conditions. Experimental sessions were conducted with groups of 4 or fewer students and lasted approximately $1 / 2 \mathrm{~h}$.

\section{Tasks and Procedure}

Four pairs of each problem type-abstract, dissimilar thematic, and similar thematic-were used. All of the abstract problems and five of the dissimilar thematic problems were identical to those used in Experiment 1. Examples of similar thematic problems are presented in Appendix B.

The order in which problem pairs were presented was counterbalanced across conditions. Within problem pairs, each problem was first for half the participants in each condition and second for the other half. For comparison purposes, the second problem of each pair was the same for participants in the similar thematic and dissimilar thematic conditions; that is, matched similar and dissimilar pairs always differed on the first problems of the pair and always shared the second problem. (Also, the response alternatives for each problem were presented in counterbalanced order across participants in each condition.)

Four pairs of problems were presented to participants; individual problems were on separate pages. The instructions were identical to those given in Experiment 1, except that no verbalization instructions were given.

The experiment was a $2 \times 3$ between-subjects factorial design. The independent variables were explanations (given or not given) and problem type (abstract, similar thematic, and dissimilar thematic). As in Experiment 1, each problem was read to the participants; in the explanation conditions, participants were read explanations after completing the first problem of each pair (i.e., after problems $1,3,5$, and 7). The dependent variables were (1) the number of completely correct selections on the second problems of the pairs; (2) the conditional probability of completely correct selections on the second problem of a pair, given completely correct selections on the first problem or given an explanation of the first problem; and (3) predominant selection patterns in each condition. Also, in the no-explanation conditions, performance on abstract versus thematic problems was examined as a planned comparison (relevant to Prediction 2b).

\section{Results}

\section{Correct Selections}

Explanation conditions. Scores on the second problem of each pair (i.e., problems 2, 4, 6, and 8) reflect transfer from the first problem of a pair and the effects of prior experience with the second problem. As can be seen in Table 2, the best performance on second problems, following explanations of the first problem, was in the abstract condition. Also, performance was better in the similar than in the dissimilar thematic condition. These patterns are in line with the predicted effects of problem type (Prediction la) and contextual similarity (Prediction $1 b$ ). A $2 \times 3$ analysis of variance partially confirmed these patterns: The predicted explanation $\times$ problem type interaction was significant $[F(2,90)=9.38, p<.001]$, as was the main effect of explanation $[F(1,90)=45.73$, $p<.001]$. Comparison between performance in the abstract and thematic conditions with explanations revealed a significant effect of problem type $[F(1,90)=3.88, p=$ $.049]$, supporting the predicted effects of problem type (Prediction 1a). However, a comparison between similar and dissimilar thematic problems in the explanation conditions did not reveal any effect of similarity on transfer between thematic problems (Prediction $1 \mathrm{~b} ; F<1$ ).

Table 2

Mean Numbers of Completely Correct Responses on the Four Even-Numbered Problems in Experiment 2

\begin{tabular}{lccc}
\hline & \multicolumn{3}{c}{ Problems } \\
\cline { 2 - 4 } Condition & Abstract & $\begin{array}{c}\text { Similar } \\
\text { Thematic }\end{array}$ & $\begin{array}{c}\text { Dissimilar } \\
\text { Thematic }\end{array}$ \\
\hline Explanation & 3.13 & 2.63 & 2.31 \\
No Explanation & .13 & 1.81 & 1.44 \\
\hline
\end{tabular}

Note-Maximum score $=4.0$. 
No-explanation conditions. The means in Table 2 also are in line with the posited effects of thematic materials (Prediction 2a) and contextual similarity without explanations (Prediction 2b). Performance on second problems was significantly better in the thematic conditions than in the abstract condition $[F(1,90)=22.16, p<.001]$, supporting Prediction $2 \mathrm{a}$ (i.e., the thematic effect). Overall, in the no-explanation conditions, participants solved approximately $46 \%$ of the problems in the similar thematic condition with no explanations, $40 \%$ in the dissimilar thematic condition with no explanations, and less than $3 \%$ in the abstract condition with no explanations. However, statistical analyses failed to support the predicted effects of contextual similarity without explanations (Prediction $2 b$ ), as transfer was no more likely to occur after similar thematic than after dissimilar thematic problems $[F(1,90)=1.29, p>.25]$.

A final analysis was undertaken to examine betweenproblem transfer across the entire series of problems. Analysis of variance of the total number of completely correct selections showed significant main effects of explanation $[F(1,90)=32.97, p<.001]$ and problem type $[F(2,90)=45.75, p<.001]$, and a significant explanation $\times$ problem type interaction $[F(2,90)=9.63, p<$ .0011 . However, both the problem type main effect and the interaction can be traced to inclusion of the abstract conditions in the analysis. The interaction indicates that over a series of problems, explanations facilitate transfer between problems of the same general type, but that this transfer is greater for abstract than for thematic problems. Furthermore, the effect of explanations appears earlier for the abstract problems; as shown in Table 3, by the fourth problem, the scores in the abstract condition with explanations had surpassed those in the thematic conditions with explanations, but by the final problem, the scores of all participants who received explanations were close to perfect.

\section{Predominant Response Patterns}

The means of predominant response patterns are presented in Table 4. Examination of these means shows that the largest difference was between the " $p$, not $q$ "

Table 3

Mean Numbers of Completely Correct Responses For Each Problem in Experiment 2

\begin{tabular}{|c|c|c|c|c|c|c|c|c|c|}
\hline \multirow[b]{2}{*}{ Explanation } & \multicolumn{8}{|c|}{ Problem Number } & \multirow[b]{2}{*}{ Overall } \\
\hline & 1 & 2 & 3 & 4 & 5 & 6 & 7 & 8 & \\
\hline \multicolumn{10}{|c|}{ Abstract Problems } \\
\hline Yes & .00 & .44 & .31 & .81 & .75 & .74 & .94 & .94 & .64 \\
\hline No & .06 & .06 & .00 & .00 & .00 & .00 & .00 & .00 & .02 \\
\hline \multicolumn{10}{|c|}{ Similar Thematic Problems } \\
\hline Yes & .25 & .38 & .50 & .56 & .31 & .69 & .81 & 1.00 & .57 \\
\hline No & .38 & .31 & .44 & .50 & .44 & .38 & .56 & .63 & .45 \\
\hline \multicolumn{10}{|c|}{ Dissimilar Thematic Problems } \\
\hline Yes & .19 & .50 & .56 & .31 & .69 & .69 & .75 & .88 & .55 \\
\hline No & .25 & .38 & .38 & .06 & .69 & .44 & .44 & .44 & .39 \\
\hline
\end{tabular}

Table 4

Mean Numbers of Predominant

Selection Patterns in Experiment 2

\begin{tabular}{lcccc}
\hline & \multicolumn{2}{c}{$\begin{array}{c}\text { Selection } \\
\text { Pattern }\end{array}$} & & \\
\cline { 2 - 3 }$\quad$ Problem Type & $p$ not $q$ & $p, q$ & Other & Unclassifiable \\
\hline Abstract & & & & \\
$\quad$ Explanation & .87 & .00 & .00 & .13 \\
$\quad$ No explanation & .00 & .56 & .31 & .13 \\
$\begin{array}{l}\text { Similar Thematic } \\
\quad \text { Explanation }\end{array}$ & .69 & .18 & .00 & .13 \\
$\quad$ No explanation & .31 & .07 & .31 & .31 \\
$\begin{array}{l}\text { Dissimilar Thematic } \\
\quad \text { Explanation }\end{array}$ & .63 & .13 & .00 & .25 \\
$\quad$ No explanation & .44 & .13 & .13 & .30 \\
\hline
\end{tabular}

Note-Maximum score $=1.0$

and " $p, q$ " selection patterns in the abstract conditions with and without explanations. An initial analysis revealed a significant three-way interaction of explanation, problem type, and selection pattern $[F(6,270)=3.98, p<.001]$.

Explanation conditions. A $2 \times 4$ analysis of variance revealed a significant problem type (abstract vs. thematic) $\times$ selection pattern interaction $[F(3,184)=3.15, p=$ .026]. For both abstract and thematic problems, the main effect of selection pattern was highly significant $[F(3,90)$ $=48.57, p<.001$, and $F(3,124)=19.48, p<.001$, respectively]. Further analyses showed that for both types of problems, the " $p$, not $q$ " selection pattern was more frequent than any other selection pattern (all $p s<.001$ ). However, comparisons between the abstract and thematic conditions in which explanations were given revealed that " $p$, not $q$ " was more frequently the predominant selection pattern in the former condition $[F(1,270)=4.4, p=$ .035]. This finding supports the prediction that transfer would be greater between abstract than between thematic problems following explanations (Prediction 1a).

However, Prediction 1b, that transfer would be greater between similar than between dissimilar thematic problems following explanations was not supported. In the thematic conditions with explanations, the problem type $\times$ selection pattern interaction was nonsignificant $(F<1)$.

No-explanation conditions. In the no-explanation conditions, a $2 \times 4$ analysis of variance showed a significant problem type $\times$ selection pattern interaction $[F(3,184)$ $=8.35, p<.001]$. The main effects of selection pattern were again significant for both abstract $[F(3,90)=$ $6.30, p<.001]$ and thematic problems $[F(3,124)=2.68$, $p=.049$, although a different pattern of results emerged for each problem type. For abstract problems without explanations, " $p, q$ " was more frequent than " $p$, not $q$ " $[F(1,90)=34.70, p<.001]$, "other" $[F(1,90)=6.98$, $p=.010]$, and "unclassifiable" selection patterns $[F(1,90)=20.20, p<.001]$. In contrast, " $p$, not $q$ " was again the most frequent selection pattern for thematic problems, but the difference from other selection patterns was significant only for the contrast with " $p, q$ " $[F(1,124)$ $=7.84, p=.006]$. The thematic effect was again in evi- 
dence, as " $p$, not $q$ " was more frequent in the thematic than abstract conditions $[F(1,270)=18.5, p<.001]$, confirming Prediction 2a.

In the thematic conditions with no explanations, the problem type $\times$ selection pattern interaction was nonsignificant $(F<1)$. Thus, Prediction $2 \mathrm{~b}$-that without explanations, more transfer would occur between similar than between dissimilar thematic problems-was not supported.

This pattern of findings using the predominant response patterns as the dependent variable was similar to that using correct selections only; transfer occurred between problems following explanations, but the relative amount of transfer was greater between abstract problems than between thematic problems. No differences were observed in the probability of transfer between pairs of similar and dissimilar thematic problems.

\section{Discussion}

The results of Experiment 2 clearly did not support the contextual similarity hypothesis offered to account for the results of Experiment 1. Participants in the similar thematic conditions did no better on second problems of pairs than did participants in the dissimilar thematic conditions.

Two important findings of Experiment 1 were replicated in this experiment. First, the thematic effect was observed again in the no-explanation conditions. Second, in the explanation conditions, transfer occurred between problems of the same general type. This transfer, however, developed earlier between abstract problems than between thematic problems.

These results apparently show that similarity, in at least a general sense, is an important factor in facilitating transfer of reasoning between problems. This conclusion is further substantiated by the results of Experiment 1; transfer occurred between problems of the same general type, but did not occur from thematic to abstract problems, even following explanations of the thematic problems. Perhaps elements from explanations of abstract problems are incorporated into a general problem solving context, from which persons are able to formulate a general problem solving rule. When abstract problems are presented, they are tied or "mapped" (Cheng et al., 1986) onto this new context, and thus are readily solvable. The elements in thematic explanations, in contrast, may not be as readily incorporated into a general context because they are tied to past experiences and, therefore, to other contexts. Thus, following explanations of thematic problems, persons may gradually form a general problem solving rule, but this rule is limited in its generality and does not apply to abstract problems.

\section{EXPERIMENT 3}

Experiment 3 was designed to test the following predictions, derived from the foregoing suggestions: (1) Participants may formulate a general rule for solving deductive problems after explanations of abstract problems; therefore, performance will be better following an initial set of abstract problems than after an initial set of thematic problems, regardless of final problem type. (2) Following explanations of abstract problems, transfer will occur to abstract problems and to thematic problems; however, the amount of this transfer will be greater when the subsequent problems are abstract. (3) After explanations of thematic problems, transfer will occur to thematic problems, but not to abstract problems.

\section{Method}

\section{Subjects}

The participants were 64 students recruited from introductory psychology classes at West Virginia University. All participants were randomly assigned across four experimental conditions.

\section{Tasks and Procedure}

Nine abstract and nine thematic problems were used; the abstract problems were similar to the abstract problems used in Experiment 2, and the thematic problems were similar to the dissimilar thematic problems (see Appendix A).

The general procedure was identical to that of the explanation conditions in Experiment 1, except that sessions were conducted with groups of 4 or fewer rather than with individuals.

The experiment was a $2 \times 2$ between-subjects factorial. The in dependent variables were initial problem type (abstract vs. thematic) and final problem type (abstract vs. thematic). The dependent variables were the total number of completely correct selections on the final four problems and predominant response patterns on the final set of problems.

\section{Correct Selections}

The mean scores of participants on the final four problems in each condition are presented in the upper portion of Table 5. As can be seen, the results supported Prediction 1, as transfer from abstract problems was greater than transfer from thematic problems. A $2 \times 2$ (initial problem type $\times$ final problem type) analysis of vari-

Table 5

Mean Numbers of Completely Correct Responses in Final in Experiment 3

\begin{tabular}{lcc} 
& \multicolumn{2}{c}{ Final Problems } \\
\cline { 2 - 3 } Initial Problems & Abstract & Thematic \\
\hline & \multicolumn{3}{c}{ Correct Selections Only* } \\
Abstract & 4.00 & 3.19 \\
Thematic & 2.00 & 2.50 \\
& Predominant Selection Patterns $\dagger$ & \\
Abstract & & \\
$p$, not $q$ & 1.00 & .80 \\
$p, q$ & .00 & .07 \\
Other & .00 & .00 \\
Unclassifiable & .00 & .13 \\
Thematic & & .62 \\
$p$, not $q$ & .37 & .00 \\
$p, q$ & .00 & .13 \\
Other & .19 & .25 \\
Unclassifiable & .44 &
\end{tabular}

\footnotetext{
*Maximum score $=4.0$. $\quad$-Maximum score $=1.0$.
} 
ance confirmed this result in that the main effect of initial problem type was significant $[F(1,60)=20.53, p<$ $.001]$. However, the initial $\times$ final problem type interaction was also significant $[F(1,60)=4.90, p=.029]$.

The significant interaction necessitated further analyses. Prediction 1 stated that transfer would be greater from initial abstract problems than from initial thematic problems, regardless of final problem type. Therefore, each condition with initial abstract problems was contrasted with each condition with initial thematic problems, using one-way analyses of variance. The findings were as follows: (1) The abstract/abstract condition differed significantly from both the thematic/abstract condition $[F(1,60)=16.97, p<.001]$ and the thematic/thematic condition $[F(1,60)=9.61, p<.01]$. (2) The abstract/thematic condition differed significantly from the thematic/abstract condition $[F(1,60)=6.01, p=.003]$, but not from the thematic/thematic condition $[F(1,60)=$ $2.02, p=.157]$. Thus, except for the last comparison, significance tests supported Prediction 1.

Prediction 2 was not fully supported by the data. Comparison between the abstract/abstract and abstract/thematic conditions revealed that the amount of transfer from an initial set of abstract problems did not differ significantly as a function of final problem type $[F(1,60)=2.79, p=$ .096], although this difference approached significance and was in the expected direction. Furthermore, examination of the means in Table 5 shows that failure to find a significant difference between these two conditions may have been due to a ceiling effect in the abstract/abstract condition.

Finally, Prediction 3 was not clearly supported. The difference between the means for the thematic/abstract and thematic/thematic conditions was not significant $[F(1,60)$ $=1.96, p=.163]$, but again the difference was in the expected direction.

\section{Predominant Response Patterns}

The means of predominant response patterns appear in the lower portion of Table 5. These means suggest that (1) between-problem transfer was greater when initial problems were abstract than when they were thematic, and (2) transfer between problems of the same type was greater than transfer between problems of different types. A $2 \times 2 \times 4$ (initial problem type $\times$ final problem type $\times$ selection pattern) analysis of variance conducted on predominant response patterns in the final problem set revealed a significant three-way interaction $[F(3,180)=$ $2.99, p=.032$ ], supporting these conclusions.

In order to test Prediction 1, further analyses were conducted to compare conditions with initial abstract problems and conditions with initial thematic problems. The frequency of the " $p$, not $q$ " selection pattern was greater in the abstract/abstract condition than in the thematic/abstract condition $[F(1,180)=68.30, p<.001]$ and the thematic/thematic condition $[F(1,180)=24.10$, $p<.001]$. It was also greater in the abstract/thematic condition than in the thematic/abstract condition $[F(1,180)$
$=31.30, p<.001]$ and the thematic/thematic condition $[F(1,180)=6.04, p=.014]$. These results provide full support for Prediction 1.

Examination of predominant response patterns also supported Prediction 2, in that " $p$, not $q$ " was more frequent in the abstract/abstract condition than in the abstract/thematic condition $[F(1,180)=7.20, p=.008]$. Prediction 3 was also supported using this scoring system, as " $p$, not $q$ " was more frequent in the thematic/thematic condition than in the thematic/abstract condition $[F(1,180)=11.10, p=.001]$. Also of note is that in each condition except the thematic/abstract condition, the " $p$, not $q$ " selection pattern was more frequent than any other selection pattern (all $p \mathrm{~s}<.001$ ). In the thematic/abstract condition, " $p$, not $q$ " was significantly more frequent than " $p, q$ " $[F(1,180)=6.86$, $p=.009]$, but not significantly more frequent than "other" and "unclassifiable" selection patterns. As the means in Table 5 indicate, in this condition the "unclassifiable" selection pattern was more frequent than any other pattern, although the difference was significant only for the contrasts with the " $p, q$ " $[F(1,180)=9.20, p=$ $.003]$ and "other" selection patterns $[F(1,180)=5.21$, $p<.025]$, not for the contrast with " $p$, not $q$ " $(F<1)$.

\section{Discussion}

Experiment 3 showed that when participants are given explanations to an initial set of abstract problems, reasoning on subsequent problems is facilitated whether the subsequent problems are abstract or thematic, and that transfer from abstract to abstract problems is greater than transfer from abstract to thematic problems. In addition, transfer is greater from an initial set of abstract problems than from an initial set of thematic problems, regardless of final problem type. Finally, transfer between problems of the same general type is greater than transfer between problems of different types.

\section{GENERAL DISCUSSION}

The combined results of the three experiments reveal several important findings. First, the thematic effect observed by others (e.g., Johnson-Laird et al., 1972) was found. Following Experiment 1, it was hypothesized that each thematic problem was related to a specific context and that similar problems had overlapping contexts. However, the results of Experiment 2 did not fully support this possibility. Instead, the solutions generated for thematic problems may reflect activation of more general pragmatic reasoning schemas (Cheng \& Holyoak, 1985; Cheng et al., 1986). Those problems that activate a permission schema are most likely to be correctly solved. Memory-cuing (Griggs, 1983) may also play a part in solving familiar thematic problems. In particular, the familiarity of the problems that are presented to participants is likely to be instrumental in determining the type of reasoning schema that is activated. In other words, 
the schema onto which problems are "mapped" (Cheng \& Holyoak, 1985) depends on how participants interpret the rules they are given. Thus, persons more familiar with the rules they are given should be able to map these rules onto the appropriate schema more readily than persons less familiar with the rules. Whether differences in rule familiarity are manifested in differential frequencies of correct solutions or in a more rapid rate of problem solving is unknown at present.

The second finding of the present research was that transfer occurred between problems, but only after explanations had been presented for initial problems. This transfer of reasoning was most apparent when the initial problems were abstract; little transfer occurred from thematic problems to abstract problems, and transfer between thematic problems was less than from abstract problems to other abstract problems or to thematic problems. Finally, although similarity between contexts generally facilitated transfer between abstract problems, it had little if any effect for thematic problems.

One possible explanation of these findings is that when participants are given explanations of initial abstract problems, the common elements of these explanations are used to form a general problem-solving context or schema. This schema may be similar to individual problems and their explanations, except that rather than being tied to a specific problem, it is applicable to any abstract problem. From this schema, participants are able to derive a general rule that they apply to subsequent problems. This general rule may be fairly straightforward (e.g., if a problem is in "if $p$, then $q$ " form, then select " $p$ " and "not $q$ ") and may be activated when similar problems (i.e., abstract problems) are mapped onto it.

The weaker transfer observed from abstract to thematic problems may be due to lower similarity between the problem types. The analogy between problem types is evidently unclear to some participants; these persons may continue to map thematic problems onto preexisting reasoning schemas. When the rules in these schemas do not correspond to the rules of formal logic, incorrect solutions result; however, when problems are mapped onto the permission schema, persons reach correct solutions. As suggested above, more familiar thematic problems may be more likely to activate preexisting schemas, inhibiting transfer; less familiar thematic problems may be less likely to activate one of these schemas and may instead activate the newly formed general problem-solving schema. For other persons, however, the applicability of the general schema to further problems seems to be more apparent, enabling them to solve further thematic and abstract problems.

In contrast, when participants are given explanations of thematic problems, because these problems are more closely tied to past experience and activate other reasoning schemas, the formation of a general problem-solving schema occurs less rapidly than with explanations of $a b-$ stract problems. On further abstract problems, this schema may still be incomplete, and participants may continue to rely on nonlogical rules such as verification (e.g., Valentine, 1985; Wason, 1966) or matching (e.g., Evans, 1977). When given abstract problems following explanations to thematic problems, only persons for whom a general schema has developed will be able to solve these problems. Most will have only incompletely developed schemas and will not be able to solve the problems.

\section{REFERENCES}

BERRY, D. C. (1983). Metacognitive experiences and transfer of logical reasoning. Quarterly Joumal of Experimental Psychology. 35A. 39-49.

Braceweil., R. J, Hidi, S. W. (1974). The solution of an inferential problem as a function of stimulus materials. Quarterly Journal of Experimental Psychology, 26, 480-488.

Cheng, P. W, Hotyoak. K. J. (1985). Pragmatic reasoning schemas. Cognitive Psychology, 17, 391-416.

Cheng, P. W. Holyoak, K. J., Nisbett, R. E., Oluver, L. M (1986). Pragmatic versus syntactic approaches to training deductive reasoning. Cognitive Psychology, 18, 293-328.

Chrostowski, J. J. Gricgs, R. A (1985). The effects of problem content, instructions, and verbalization procedure on Wason's selection task. Current Psychological Research \& Reviews, 4, 99-107.

Cox, J. P., GRIGGs, R. A. (1982). The effect of experience on performance in Wason's selection task. Memory \& Cognition, 10, 496-502.

Evans, 3. ST. B. T. (1972), On the problem of interpreting reasoning data: Logical processes and approaches. Cognition, 1, 373-384.

Evans. J. St. B. T. (1977). Toward a statistical theory of reasoning Quarterly Joumal of Experimental Psychology, 29, 193-199.

Gilhooly, K. J., FAlConer, W. A. (1974). Concrete and familiar terms and the relations in testing a rule. Quarterly Joumal of Experimental Psychology, 26, 355-359.

GrIGGS, R. A. (1983). The role of problem content in the selection task and the THOG problem. In J. St. B. T. Evans (Ed.), Thinking and reasoning: Psychological approaches (pp. 16-43). London: Routlodge \& Kegan Paul

GrJGGS, R. A. (1984). Memory-cueing and instructional effects on Wason's selection task. Current Psychological Research \& Reviews, 3. 3-10.

Griggs, R. A., \& Cox, J. R. (1982). The elusive thematic-materials effect in Wason's selection task. British Journal of Psychology, 73, 407-420.

Johnson-Laird, P. N., Lengrezi, P., Lengrezi, M. S. (1972). Reasoning and a sense of reality. British Joumal of Psychology, 24. $395-400$.

Lunzer, E. A., Harrison, C., Davey, M. (1972). The four-card selection problem and generality of formal reasoning. Quanerly Journal of Experimental Psychology, 24, 326-339.

Manktelow, K. I., Evans, J. (1979). Facilitation of reasoning by realism: Effect or noneffect. British Journal of Psychology, 70, 477-488.

REICH, S. \&UTh, P. (1982). Wason's selection task: Verification, falsification, and matching. British Joumal of Psychology, 73, 395-405.

VALENTINE, E. R. (1985). The effect of instructions on performance in the Wason selection task. Current Psychological Research \& Reviews, 4, 214-223.

VAN DUYNE, P. C. (1974). Reasoning and linguistic complexity in reasoning. British Journal of Psychology, 65, 59-67.

WAson, P. C. (1966). Reasoning. In B. Foss (Ed.), New horizons in psychology. Harmondworth, England: Penguin Books.

WAsON, P. C. (1968). Reasoning about a rule. Quarterly Joumal of Experimental Psychology, 20, 63-71. 
WAsON, P. C. (1983). Realism and rationality in the selection task. In J. St. B. T. Evans (Ed.), Thinking and reasoning: Psychological approaches (pp. 44-75). London: Routledge and Kegan Paul.

WAson, P. C., Shaptro, D. (1971). Natural and contrived experience in a reasoning problem. Quarterly Journal of Experimental Psychology, 23, 63-71.

Yachanin, S. A., \& Tweney, R. D. (1982). The effect of thematic content on cognitive strategies in the four-card selection task. Bulletin of the Psychonomic Sociery, 19, 87-90.

\section{APPENDIX A}

\section{Examples of Problems Used in Experiments 1 and 3}

\section{Examples of Abstract Problems}

(1) Your task on the following problem is to determine whether or not this rule is in effect:

"If $1 / 2$ is on one side of a card, then Helium must be on the other side of the card."

This rule may or may not be in effect.

[The four cards you have in front of you each has two pieces of information on it. On one side of each card, there is a fraction, and on the other side of each card, there is a chemical element. For two of these cards, you can see the fraction on one side of the card, but you cannot see the chemical element on the other side of the card. For the other two cards, you can see the chemical element on one side of the card, but you cannot see the fraction on the other side of the card.

Your task is to decide what additional information you would need to decide whether or not the above rule is in effect. For which of these four cards would the information on the other side of the card help you test whether or not this rule is in effect?

Be sure to select all those cards that you need in order to make your decision, but only those cards that you definitely need.]*

$$
\frac{\text { CARDS }}{\frac{\mathrm{A}}{1 / 2}} \underset{\text { Oxygen }}{\frac{\mathrm{B}}{\text { Helium }}} \frac{\mathrm{D}}{3 / 4}
$$

*Note: The bracketed section is presented here in order to illustrate differences in this section between abstract and thematic problems. As with thematic problems, this section can be reconstructed readily; hence, it is omitted from the remaining problems.

(2) Your task on the following problem is to determine whether or not this rule is in effect:

"If Chair is on one side of a card, then Blond must be on the other side of the card."

CARDS
$\frac{\text { A }}{\text { Brunette }} \quad \frac{\text { B }}{\text { Blond }} \quad \frac{\text { D }}{\text { Table }} \quad \frac{\text { Chair }}{4}$

(3) Your task on the following problem is to determine whether or not this rule is in effect:

"If Doctor is on one side of a card, then Pistachio must be on the other side of the card."

$\frac{\text { CARDS }}{\frac{\mathrm{A}}{\text { Doctor }}} \frac{\mathrm{B}}{\text { Mailman }} \frac{\mathrm{C}}{\text { Peanut }} \quad \frac{\mathrm{D}}{\text { Pistachio }}$

(4) Your task on the following problem is to decide whether or not this rule is in effect:

"If Salt is on one side of a card, then Pink must be on the other side of the card."

CARDS
$\frac{\text { A }}{\text { Pink }} \quad \frac{\text { B }}{\text { Purple }} \frac{\text { D }}{\text { Pepper }}$

(5) Your task on the following problem is to determine whether or not this rule is in effect:

"If Corvette is on one side of a card, then Catholic must be on the other side of the card."

\begin{tabular}{|c|c|c|c|}
\hline \multicolumn{4}{|c|}{ CARDS } \\
\hline A & B & $\mathrm{C}$ & D \\
\hline $\operatorname{rans} A m$ & Hindu & Catholic & Corvette \\
\hline
\end{tabular}

(6) Your task on the following problem is to determine whether or not this rule is in effect:

"If Tuna is on one side of a card, then Coffee must be on the other side of the card."

\begin{tabular}{cccc}
\multicolumn{4}{c}{ CARDS } \\
$\frac{\text { A }}{\text { Goldfish }} \quad \frac{\text { B }}{\text { Coffee }}$ & $\frac{\text { C }}{\text { Tuna }}$ & $\frac{\text { D }}{\text { Coke }}$
\end{tabular}

(7) Your task on the following problem is to determine whether or not this rule is in effect:

"If Winter is on one side of a card, then Jet must be on the other side of the card."

\begin{tabular}{cccc}
\multicolumn{4}{c}{ CARDS } \\
$\frac{\mathrm{A}}{\text { Biplane }}$ & $\frac{\mathrm{B}}{\text { Winter }}$ & $\frac{\mathrm{C}}{\text { Jet }}$ & $\frac{\mathrm{D}}{\text { Spring }}$
\end{tabular}

(8) Your task on the following problem is to determine whether or not this rule is in effect:

"If there is an A on one side of a card, then there must be a 3 on the other side of the card."

CARDS

$\overline{\mathrm{A}} \overline{\mathrm{D} "} \quad \frac{\mathrm{B}}{" 3 "} \quad \frac{\mathrm{C}}{" 7 "} \quad \frac{\mathrm{D}}{" \mathrm{~A} "}$

(9) Your task on the following problem is to determine whether or not this rule is in effect:

"If Chicago is on one side of a card, then Cat must be on the other side of the card."

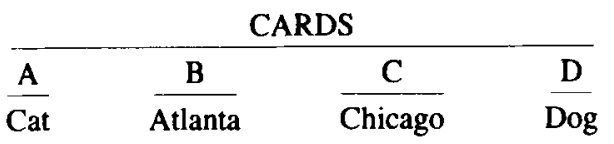

\section{Thematic Problems}

(1) Imagine you are a police officer, on duty, walking through a local bar. It is your job to ensure that the local drinking laws are in effect in this bar. One such law is:

"If a person is drinking beer, then that person must be at least 19 years old." 
The rule may or may not be in effect.

[The four cards you have in front of you each represents one of the people drinking at the bar. There are two pieces of information about a person on a card: a person's age is on one side of a card, and what the person is drinking is on the other side of the card. For two of the people, you can see their age but you cannot see what they are drinking. For the other two people, you can see what they are drinking, but you cannot see their age.

Your task is to decide what additional information you would need in order to decide whether or not the drinking law is in effect. For which of these four people would the information on the other side of the card help you decide whether or not the bar is observing the law?

Be sure to select all those cards that you need in order to make your decision, but only those cards that you definitely need.]*

\begin{tabular}{cccc}
\multicolumn{4}{c}{ PERSON } \\
\hline $\mathrm{A}$ & $\mathrm{B}$ & $\mathrm{C}$ & $\mathrm{D}$ \\
\cline { 2 - 3 } $\begin{array}{c}\text { Drinking } \\
\text { Beer }\end{array}$ & $\begin{array}{c}21 \text { Years } \\
\text { Old }\end{array}$ & $\begin{array}{c}\text { Drinking } \\
\text { Coke }\end{array}$ & $\begin{array}{c}16 \text { Years } \\
\text { Old }\end{array}$
\end{tabular}

*Note: Brackets indicate a portion of each problem that was nearly identical across problems and, therefore, is omitted from subsequent problems. With the exception of the words used to represent " $p$," " $q$," " not $p$," and "not $q$," this section is the same for each problem and thus is easily reconstructed.

(2) Imagine you are the dean at a local university examining students' academic performance. One of your jobs is to make sure that certain rules concerning students' grades are in effect. One such rule is:

"If a person plays on the basketball team, then that person must have at least a $\mathrm{C}$ average."

\begin{tabular}{|c|c|c|c|}
\hline \multicolumn{4}{|c|}{ STUDENT } \\
\hline A & B & $\mathrm{C}$ & $\mathrm{D}$ \\
\hline "D" & $\overline{~ " A "}$ & $\begin{array}{c}\text { Plays } \\
\text { Basketball }\end{array}$ & $\begin{array}{c}\text { Does Not } \\
\text { Play Basketball }\end{array}$ \\
\hline
\end{tabular}

(3) Imagine you are a librarian working on the returned book files. Part of your job concerns enforcing certain rules regarding these files. One such rule is:

"If a person returns a book late, then that person must be fined."

\begin{tabular}{cccc}
\multicolumn{3}{c}{ PERSON } \\
$\frac{\mathrm{A}}{\text { Returned Late }} \quad \frac{\mathrm{B}}{\text { Not Fined }}$ & $\frac{\mathrm{C}}{\text { Fined }}$ & $\frac{\mathrm{D}}{\text { On Time }}$
\end{tabular}

(4) Imagine you are a highway patrolman monitoring the traffic passing by you. One of your jobs is to make sure that when a person violates a traffic law, he is punished according to certain laws. One of these laws is:

"If a person is speeding, then that person must be ticketed."

\begin{tabular}{cccc}
\multicolumn{4}{c}{ PERSON } \\
$\frac{\mathrm{A}}{\text { Not Speeding }}$ & $\frac{\mathrm{B}}{\text { Ticketed }}$ & $\frac{\mathrm{C}}{\text { Speeding }}$ & $\frac{\mathrm{D}}{\text { Not Ticketed }}$
\end{tabular}

(5) Imagine you are a state official investigating the local voting records. One of your jobs includes checking these records to make sure that local voters meet certain registration requirements. One such requirement is:
"If a person is allowed to vote, then that person must be registered."

\begin{tabular}{ccc}
\multicolumn{3}{c}{ VOTER } \\
$\frac{\mathrm{A}}{\text { Registered }} \frac{\mathrm{B}}{\text { Not Registered }} \frac{\mathrm{C}}{\text { Did Not Vote }}$ & $\frac{\mathrm{D}}{\text { Voted }}$
\end{tabular}

\section{Examples of Explanations to Abstract and Thematic Problems}

Explanation to the fraction-chemical problem (abstract). The correct answer is to turn over both the " $1 / 2$ " and the "Oxygen" cards. The rule states that if there is a $1 / 2$ on one side of a card, then Helium must be on the other side of that card. The " $1 / 2$ " " card must be turned over because the truth of the rule is determined by the chemical element on the other side of this card. If the element on the other side of this card is any element other than helium, then the rule cannot be in effect. Likewise, the "Oxygen' card must be turned over because, according to the rule, Oxygen cannot be on the other side of a card that has $1 / 2$ on it. If a card with Oxygen on one side has $1 / 2$ on the other side of it, the rule cannot be in effect. In order to see if the "Oxygen" card has $1 / 2$ on the other side of it, one must turn this card over. The " $3 / 4$ " card does not have to be turned over because any chemical could be on the other side of this card and the rule could still be in effect; that is, the chemical that appears on the other side of this card does not affect the truth of the rule, so the information on the other side of this card is irrelevant. The "Helium' card does not have to be turned over either. Because the rule is not reversible, a card with Helium on one side does not have to have $1 / 2$ on the other side of it; either $1 / 2$ or $\#$ could be on the other side of this card and the rule could still be in effect.

Explanation to the drinking problem (thematic). The correct answer is to turn over both the "Drinking Beer" and the "16 Years Old" cards. The law states that if a person is drinking beer, then that person must be at least 19 years old. The "Drinking Beer" card must be turned over because the truth of the law is determined by whether or not that person is over 19 years old. If the age on the other side of this card is under 19 , then the law cannot be in effect. Likewise, the " 16 Years Old" card must be turned over because, according to the law, this person cannot be drinking beer. If a person who is 16 years old is drinking beer, then the law cannot be in effect. In order to see if the 16-year-old person is drinking beer, one must turn this card over. The "Drinking Coke' card does not have to be turned over because any age could be on the other side of this card and the law could still be in effect; that is, the age of a person drinking Coke does not affect the truth of the law, so the information on the other side of this card is irrelevant. The "21 Years Old" card does not have to be turned over either. Because the law is not reversible, a person who is 21 does not have to be drinking beer; either drinking beer or drinking Coke could be on the other side of this card and the law could still be in effect.

\section{APPENDIX B \\ Examples of Similar Thematic Problems}

(1a) Imagine you are a police officer, on duty, walking through a local bar. It is your job to ensure that the local drinking laws are in effect in this bar. One such law is:

"If a person is drinking beer, then that person must be at least 19 years old."

This law may or may not be in effect. 
PERSON

$\begin{array}{ccccc}\frac{A}{\text { Drinking }} & \frac{B}{\text { Beer }} & \begin{array}{c}22 \text { Years } \\ \text { Old }\end{array} & \begin{array}{c}\text { Drinking } \\ \text { Coke }\end{array} & \begin{array}{c}16 \text { Years } \\ \text { Old }\end{array}\end{array}$

(1b) As an on-duty police officer, there are also other laws that you are to keep in effect. Another of these laws is:

"If a person is drinking beer outside of a bar, then that person must be fined."

This law may or may not be in effect.

\section{PERSON}

\begin{tabular}{cccc}
$\frac{\mathrm{A}}{\text { Drinking }}$ & Fined & $\begin{array}{c}\text { Drinking } \\
\begin{array}{c}\text { Beer } \\
\text { Beer } \\
\text { Outside }\end{array}\end{array}$ & $\begin{array}{c}\text { Not } \\
\text { Fined }\end{array}$ \\
\cline { 4 - 5 } & & & Pined
\end{tabular}

(2a) Imagine you are a librarian working on the returned book files. Part of your job concerns enforcing certain rules regarding these files. One such rule is:

"If a person returns a book late, then that person must be fined."
This rule may or may not be in effect.

\begin{tabular}{cccc}
\multicolumn{4}{c}{ PERSON } \\
$\begin{array}{c}\text { Returned } \\
\text { Late }\end{array}$ & $\begin{array}{c}\text { B } \\
\text { Fined }\end{array}$ & $\begin{array}{c}\text { Returned } \\
\text { On } \\
\text { Time }\end{array}$ & Fined \\
& Fined &
\end{tabular}

(2b) As a librarian, there are also other rules you must keep in effect. Another of these rules is:

"If a person checks a book out, then that person must have a library card."

This rule may or may not be in effect.

\begin{tabular}{|c|c|c|c|}
\hline \multicolumn{4}{|c|}{ PERSON } \\
\hline A & B & C & D \\
\hline Not & Library & No & Checking \\
\hline $\begin{array}{l}\text { Checking } \\
\text { Book Out }\end{array}$ & Card & Card & Book Out \\
\hline
\end{tabular}

(Manuscript received February 17, 1987; revision accepted for publication May 24, 1988.) 